\title{
Dermal microfilariae of dogs, jackals and cats in different regions of Iran
}

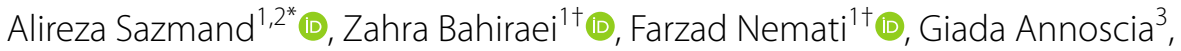 \\ Marcos Antonio Bezerra-Santos ${ }^{3}$ [D, Hassan Nayebzadeh ${ }^{4}$ (D) Amir Masoud Salemi ${ }^{5}$ (D), \\ Seyed Mahmoud Mousavi ${ }^{5}$, Seyed Mahmoud Sadjjadi ${ }^{5,6}$ [D and Domenico Otranto ${ }^{1,3}$ (B)
}

\begin{abstract}
Background: Due to the complexity of retrieving skin-dwelling microfilariae, filarioids of dogs presenting dermal microfilariae (e.g. Cercopithifilaria spp., Onchocerca lupi) are relatively unknown compared to Dirofilaria spp. and Acanthocheilonema spp. whose microfilariae circulate in the blood. Although Cercopithifilaria spp. and O. lupi filarioids are distributed worldwide, there is a paucity of information on their occurrence in Iran. The aim of this study was to investigate these filarioids in a large population of dogs from different regions of Iran.
\end{abstract}

Methods: From October 2018 to September 2020, skin biopsies were obtained from dogs housed in shelters $(n=557)$ and privately owned dogs $(n=26)$ in seven provinces of Iran (Hamedan, Kermanshah, Yazd, Mazandaran, Khuzestan, Lorestan, Esfahan), as well as from three road-killed jackals (Canis aureus) and three cats (Felis catus) in Hamedan province. The skin biopsies were first soaked in saline solution at room temperature overnight, and examined for dermal microfilariae under the microscope. Positive skin specimens and sediments were tested by PCR for a 304-bp region of the mitochondrial cytochrome c oxidase subunit 1 (cox1) gene and amplicons were sequenced.

Results: Microfilariae of Cercopithifilaria spp. were found in skin biopsies of 32 of the 583 (5.5\%) dogs tested, with infection rates of up to $25 \%$ in Kermanshah. No microfilariae were recovered from skin biopsy samples collected from dogs in Khorramabad and Ahvaz, nor from the examined jackals and cats. None of the privately owned dogs were found to be infected. Morphologic and morphometric characteristics of the microfilariae were consistent with $C$. bainae. Eighteen skin samples were positive for the cox 1 gene, of which 15 sequences showed a nucleotide identity of $100 \%$ and three of $93.4 \%$ with the reference sequence of C. bainae available in GenBank (haplotype I; GenBank accession number: JF461457).

Conclusions: The data from this study broadens current knowledge on the geographical distribution of C. bainae in dogs in Middle Eastern countries. Further studies on different wild canine species in the country (e.g. jackal, fox, wolf) could provide further information on the epidemiology of these filarioids. A particular focus should be put on zoonotic O. lupi given the reports of its presence in human patients from this country.

Keywords: Cercopithifilaria bainae, Dermal microfilariae, Onchocerca lupi, Dog, Neglected, Vector-borne

\footnotetext{
*Correspondence: alireza.sazmand@basu.ac.ir

†Zahra Bahiraei and Farzad Nemati contributed equally to this work

1 Department of Pathobiology, Faculty of Veterinary Science, Bu-Ali Sina University, 6517658978 Hamedan, Iran

Full list of author information is available at the end of the article
}

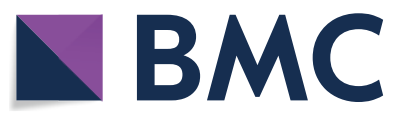

(c) The Author(s) 2022. Open Access This article is licensed under a Creative Commons Attribution 4.0 International License, which permits use, sharing, adaptation, distribution and reproduction in any medium or format, as long as you give appropriate credit to the original author(s) and the source, provide a link to the Creative Commons licence, and indicate if changes were made. The images or other third party material in this article are included in the article's Creative Commons licence, unless indicated otherwise in a credit line to the material. If material is not included in the article's Creative Commons licence and your intended use is not permitted by statutory regulation or exceeds the permitted use, you will need to obtain permission directly from the copyright holder. To view a copy of this licence, visit http://creativecommons.org/licenses/by/4.0/. The Creative Commons Public Domain Dedication waiver (http://creativeco mmons.org/publicdomain/zero/1.0/) applies to the data made available in this article, unless otherwise stated in a credit line to the data. 


\section{Graphical Abstract}

\section{Dermal microfilariae of dogs, jackals and cats in different regions of Iran}

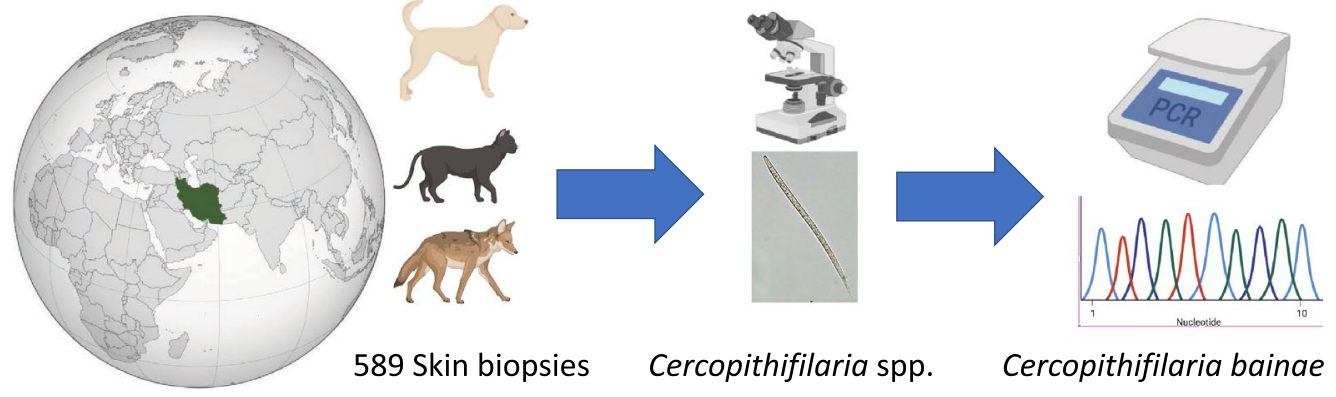

\section{Background}

Until 15 years ago, filarial nematodes releasing microfilariae into the haematic circulation, such as Dirofilaria immitis, Dirofilaria repens, Acanthocheilonema reconditum, Acanthocheilonema dracunculoides and Brugia malayi, were considered to be the most prevalent filarioids affecting dogs [1]. Conversely, those which present dermal microfilariae, such as Cercopithifilaria spp. and Onchocerca lupi, have been largely neglected by parasitologists and veterinary practitioners mainly due to the difficulties in retrieving microfilariae in the skin and to the limited number of clinical conditions they cause [2, 3]. Members of the genus Cercopithifilaria are parasites of cercopithecus monkeys, ruminants, rodents, lagomorphs, marsupials, monotremes and carnivores $[4,5]$. In the past decade, three of the 29 species of Cercopithifilaria (i.e. Cercopithifilaria bainae, Cercopithifilaria grassii and Cercopithifilaria sp. II sensu Otranto et al. 2013) have been reported in domestic dogs (Canis lupus familiaris) and red foxes (Vulpes vulpes) of Europe and the Americas $[6,7]$. Infections with Cercopithifilaria spp. are generally considered to be minimally pathogenic as most of the infected dogs do not present physical abnormalities that could be associated to the presence of microfilariae in the skin. However, dermatitis [7, 8], diffused chronic polyarthritis without any other apparent cause [9] and gross skin lesions [10] have been reported in infected dogs. Recently, an infected dog with a giant cutaneous cyst in the lumbosacral region with numerous microfilariae of C. bainae was also reported [11], suggesting a further pathogenic role of this species in infected dogs. Rhipicephalus sanguineus sensu lato (s.l.) is considered to be the competent biological vector of these nematodes [12,13] although DNA of Cercopithifilaria spp. has been also detected in Rhipicephalus turanicus and different genogroups within $R$. sanguineus s.l. from different countries in Europe, Africa, Asia and Australia [14].

Microfilariae of the zoonotic nematode Onchocerca lupi, which infects dogs, wolves, cats, coyotes and humans, can also be detected in skin biopsies of the infected carnivores [15]. Infected dogs may display acute or chronic ocular diseases [16] or be completely asymptomatic due to the localisation of adult nematodes in the retrobulbar space of the eye [17]. The parasite's life-cycle, the incubation time and the prepatent period are not fully understood, but black flies of the genus Simulium are suspected to be involved as intermediate hosts [15]. Since the first report of zoonotic infection by $O$. lupi in humans [18], infection has been diagnosed in more than 20 patients in Iran, Turkey, Tunisia, Greece, Hungary, Germany and the USA [19]. Human patients manifest an invasive disease featured by spinal, orbital, ocular and subdermal nodules [20-22].

Canine and feline infection with dermal microfilariae has been reported from different European and American countries [23]. However, although human infection with $O$. lupi has been confirmed in Iran and neighbouring Turkey $[18,22,24]$ no information is available on $O$. lupi infection in dogs.

Dogs affected by Cercopithifilaria spp. and O. lupi sometimes do not display any clinical signs $[25,26]$. In the context of this background, together with the presence of $R$. sanguineus s.l. (i.e. vectors of Cercopithifilaria spp.) in Iran [27-31], we decided to investigate dermal microfilariosis in dogs from different regions of the country, aiming to improve scientific knowledge on the distribution of these little known filarioids.

\section{Methods \\ Study area}

The study was carried out in seven provinces in Iran (i.e. Hamedan, Kermanshah, Yazd, Mazandaran, Khuzestan, Lorestan and Esfahan (Fig. 1). Most of the samples 


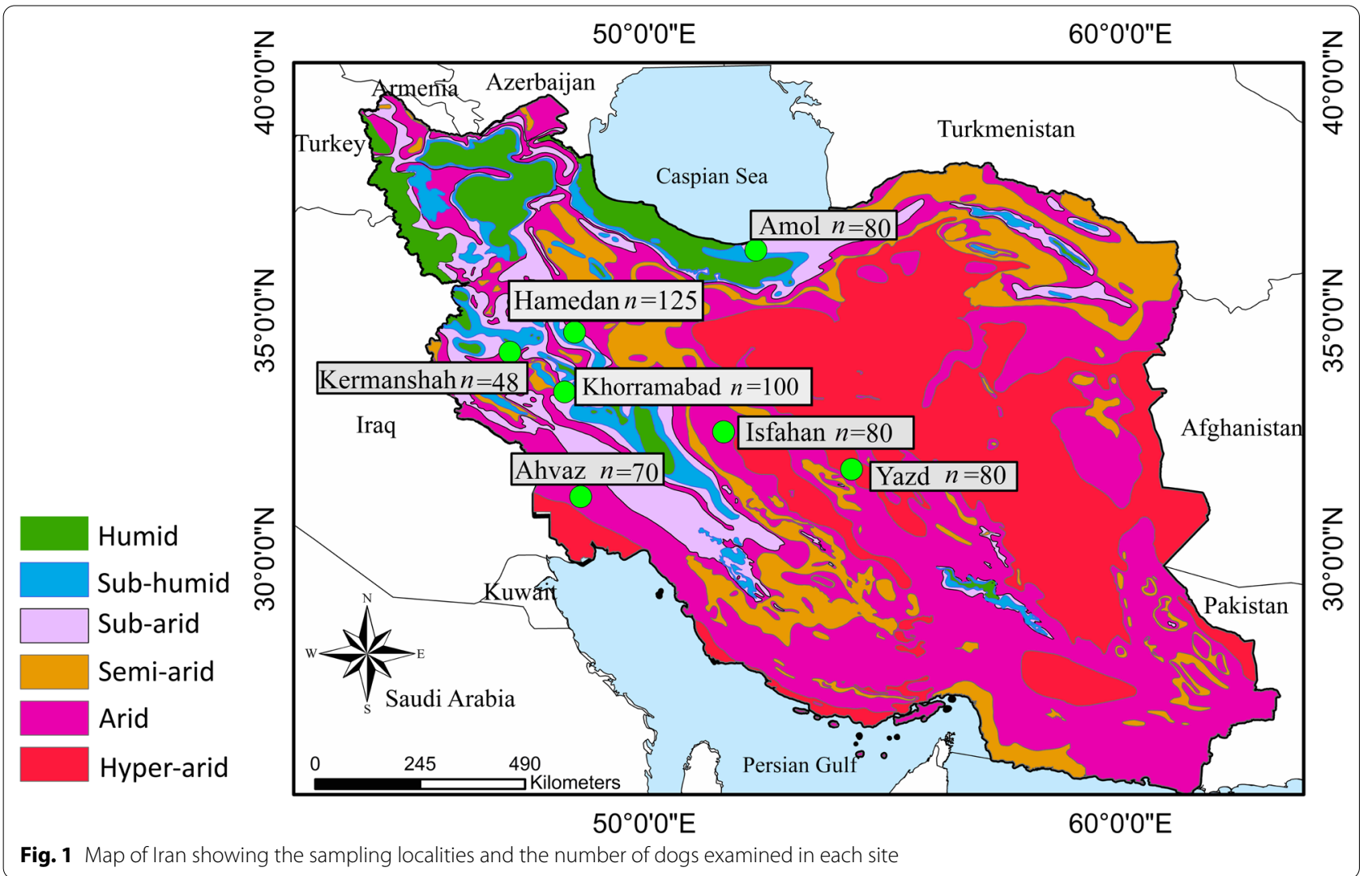

(537/538; 92.1\%) were taken from animal shelters that had been built by non-governmental organisation (NGOs) to feed stray dogs. Dogs in these shelters had been rescued from all of the seven provinces (Fig. 2).

\section{Collection of specimens and microscopic examination}

From October 2018 to September 2020, 583 dogs aged > 6 months (537 dogs from the chosen shelters and 46 privately owned dogs) were sampled at seven sampling sites (Fig. 1). Clinical examination was performed, and anamnestic data were recorded for each dog sampled; the dogs sampled had not been treated with endoparasiticides for at least 1 month prior to the sampling. During the study period, three road-killed jackals (Canis aureus) and three cats (Felis catus) in Hamedan were also biopsied.

Skin biopsies (about $0.5 \times 0.5 \times 0.5 \mathrm{~cm}$ ) were taken using disposable scalpels from the inter-scapular region and soaked in $1 \mathrm{ml}$ saline solution $(\mathrm{NaCl} 0.9 \%)$ at room temperature for $10 \mathrm{~h}$ or overnight. A drop of sediment from the soaked skin sample was then placed on a glass slide and observed under the light microscope (2 fields of $18 \times 18$-mm coverslip each) at a magnification of $\times 100$. Dermal microfilariae were counted and identified based on morphological features described previously [10, 25].
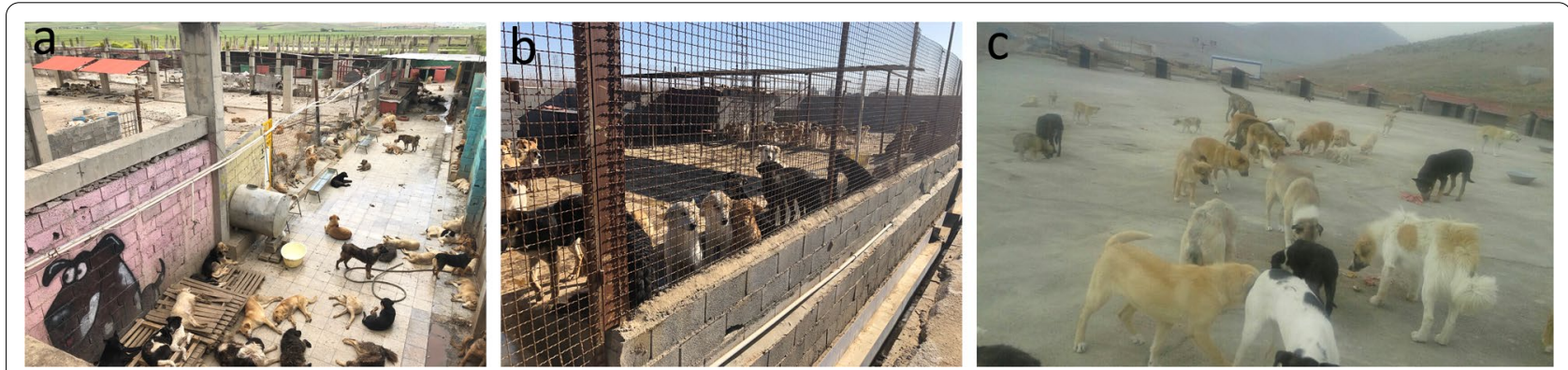

Fig. 2 Shelters in Kermanshah (a), Yazd (b) and Lorestan (c) provinces where dogs were sampled 
Photomicrographs were taken of microfilariae using an Olympus CX41 microscope (Olympus Corp., Tokyo, Japan) equipped with a DP25 camera, and the microfilariae measured. For permanent mounting of microfilariae, the coverslips of positive slides were removed, and then both slides and coverslips were fixed with methanol, then air-dried and stained with Giemsa stain as described previously [32]. Upon diagnosis of microfilariae by microscopy, the skin tissue was transferred into clean microtubes and stored at $-20^{\circ} \mathrm{C}$ for molecular analysis.

\section{Molecular procedures}

Genomic DNA was extracted from all skin samples and sediments using a commercial kit (DNeasy ${ }^{\circledR}$ Blood \& Tissue kit; Qiagen, Hilden, Germany), according to the manufacturer's instructions. A 304-bp region of the mitochondrial cytochrome $c$ oxidase subunit 1 ( $\operatorname{cox} 1)$ gene was amplified using the CbCox1F/NTR primer pair [33]. Amplicons were visualised by electrophoresis in a $2 \%$ agarose gel stained with GelRed (VWR International PBI, Milan, Italy) on a GelLogic 100 gel documentation system (Kodak, Rochester, NY, USA). Amplicons were then purified and sequenced in both directions using the same primers as those for the PCRs, by the Big Dye Terminator v.3.1 Cycle Sequencing Kit in a 3130 Genetic Analyzer (Applied Biosystems, Thermo Fisher Scientific, Foster City, CA, USA). Sequences were edited and compared to those available in the GenBank ${ }^{\mathrm{TM}}$ dataset by Basic Local Alignment Search Tool (BLAST) analysis (https:// blast.ncbi.nlm.nih.gov/Blast.cgi). Phylogenetic relationships were inferred using the Maximum Likelihood (ML) method based on the Tamura-Nei model [34], with the Gamma distribution $(+\mathrm{G})$ used to model evolutionary rate differences among sites selected by the best-fit model [35]. Evolutionary analysis was conducted on 1000 bootstrap replications using MEGA5 software [36]. A homologous sequence from Litosomoides yutajensis (GenBank accession number: AJ544869) was used as the outgroup.

\section{Statistical analysis}

Exact binomial 95\% confidence intervals (CIs) were established for proportions. The chi-square $\left(\chi^{2}\right)$ test was used to compare proportions, with a probability value $<0.05$ regarded as statistically significant. Analyses were performed using Microsoft Office Excel ver. 16.37 (Microsoft Corp., Redmond, WA, USA).

\section{Results}

Of the 583 dogs from whom skin biopies were taken and examined, 32 (5.5\%; 95\% CI 5.47-5.51) from five regions $(n=12$ Kermanshah; $n=7$ from Hamedan; $n=7$ from Yazd; $n=3$ from Amol; $n=3$ from Esfahan) were positive for microfilariae. Biopsies taken from dogs sampled in Ahvaz and Khorramabad $(n=170)$ all tested negative for microfilariae based on microscopic examination. None of the privately owned dogs nor the jackals and cats were found to be infected. No statistical association was found between Cercopithifilaria spp. infection in dogs and sex $\left(\chi^{2}=1.43, d f=1\right.$, $P=0.23)$ or age $\left(\chi^{2}=0.49, d f=3, P=0.92\right)$ (Table 1 ). At clinical examination, 69 dogs (11.8\%) showed ocular clinical signs, such as mucosal discharges, conjunctivitis and keratitis or ulcers.

All of the microfilariae were identified as C. bainae, with mean length and width of 172.32 and $6.77 \mu \mathrm{m}$, respectively, a rounded head and a short dorsoventrally flattened body (Fig. 3, Additional file 1: Video S1). A maximum number of five microfilariae were counted in one sediment. Molecular testing showed that 18 skin samples (56.2\%) were positive for the cox 1 gene. Of these positive samples, 15 sequences showed a nucleotide identity of $100 \%$ with the reference sequence of C. bainae haplotype I (GenBank accession number: JF461457) and the remaining three showed $93.4 \%$

Table 1 Number and percentage of dogs positive for Cercopithifilaria spp. $(n=583)$ according to sex, age and sampling area

\begin{tabular}{ll}
\hline Variables & Cercopithifilaria infection \\
\hline Sex & \\
Male & $16 / 207(7.7 \% ; 7.69-7.77)$ \\
Female & $14 / 368(3.8 \% ; 3.78-3.82)$ \\
Unknown & $2 / 8(25 \%)$ \\
Age (years) & \\
$<1$ & $2 / 49(4.1 \% ; 4.02-4.14)$ \\
$1-3$ & $19 / 328(5.8 \% ; 5.77-5.82)$ \\
$3-5$ & $5 / 105(4.8 \% ; 4.72-4.80)$ \\
$>5$ & $5 / 96(5.2 \% ; 5.16-5.25)$ \\
Unknown & $1 / 5(20 \%)$ \\
Geographical origin & \\
Kermanshah & $12 / 48 \%(25 ; 24.87-25.13)$ \\
Yazd & $7 / 80(8.7 \% ; 8.69-8.81)$ \\
Hamedan & $7 / 125(5.6 \% ; 5.2-6.0)$ \\
Amol & $3 / 80(3.7 \% ; 3.71-3.79)$ \\
Esfahan & $3 / 80(3.7 \% ; 3.71-3.79)$ \\
Ahvaz & $0 / 70$ \\
Khorramabad & $0 / 100$ \\
Total & $32 / 583(5.5 \% ; 5.47-5.51)$ \\
\hline
\end{tabular}

${ }^{a}$ Infection values are presented as the number of positive skin samples/total number of skin samples tested, with the percentage and $95 \%$ confidence interval given in parentheses 


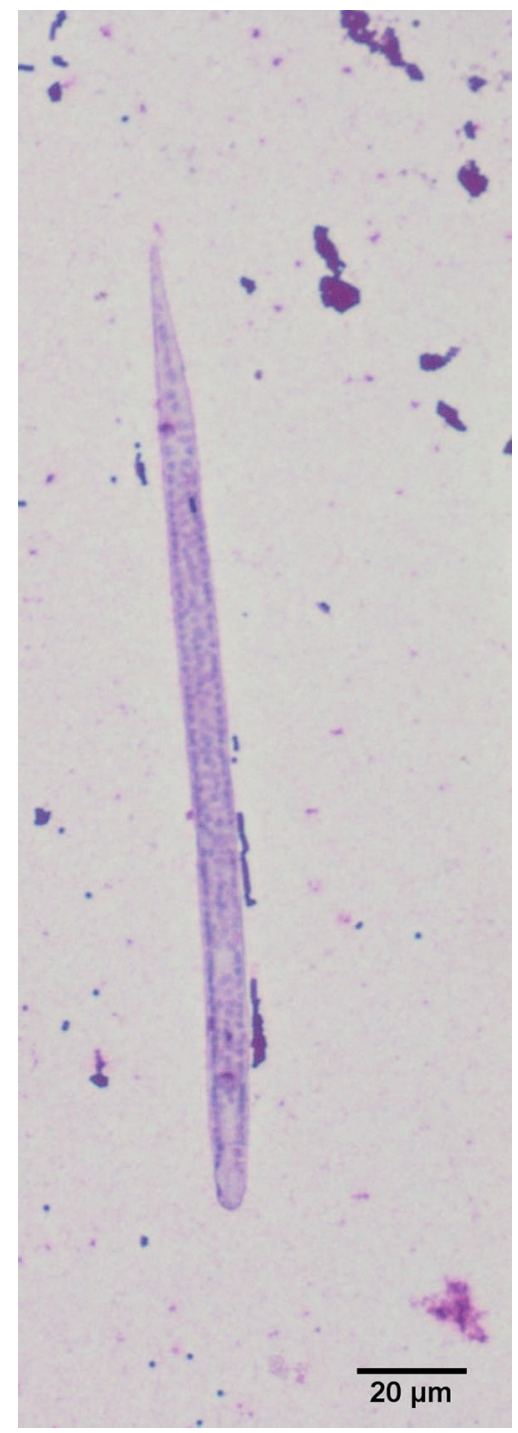

Fig. 3 Microfilaria of Cercopithifilaria bainae stained with Giemsa. Note the rounded head, short body and tip-pointed tail of the microfilaria (scale bar: $20 \mu \mathrm{m}$ )

similarity (Fig. 4). The nucleotide sequences of Cercopithifilaria sp. identified in this study were deposited in GenBank (accession number: OL873253).

\section{Discussion}

Our survey of $C$. bainae infection in dogs from different regions of Iran indicates that this filarioid is more widespread than previously thought. However, the presence of Cercopithifilaria spp. in dogs from Iran could be also inferred by the fact that $R$. sanguineus s.l. is distributed in that region [27]. More specifically, up to $5.5 \%$ of the dogs tested were infected with C. bainae. In previous studies, infection rates of $9.4 \%$ and $13.9 \%$ were reported in Italy [1, 37], 21.6\% in Spain [1], 4.3\% and $13.6 \%$ in Greece [1, 26], $9.8 \%$ in Portugal [38], 2.6\% in Romania [39], 1\% in Brazil [40] and 2.6\% in USA [41]. A similar variation was found in our survey, with the infection rates in dogs varying according to the study regions, with the highest prevalence of infection (25\%) in Kermanshah and no cases in Ahvaz and Khorramabad. This pattern is similar to that observed in dogs from Italy, where the prevalence of $C$. bainae ranged from $7.7 \%$ in Sardinia to $13.3 \%$ in Sicily $[1,37]$. The prevalence of this filarioid has also been reported to vary in Greece, between $2 \%$ and $30.8 \%$ depending on the sampling location [26]. Of note, infection in the sampled dogs in the different regions could not be associated with climate, elevation above sea level or average annual precipitation (Table 2). It has been hypothesised that Cercopithifilaria spp. might be more common in warmer areas [39].

In this study, C. bainae was the only filarioid species detected. To date, co-infection of C. bainae with other Cercopithifilaria spp. has been reported in animal populations of Italy with Cercopithifilaria sp. II and C. grassi [10, 37], in Greece with C. grassii [10, 26] and in Spain and Portugal with C. grassii and Cercopithifilaria sp. II [38]. Despite other Cercopithifilaria spp. not being detected in our study, they could be present in domestic dogs and/or wild canids of Iran. For example, DNA of C. grassi has been identified in one Rhipicephalus sp. III tick in the neighbouring country of Pakistan [14].

There was co-infection with other tick-borne pathogens in 17 of 32 dogs infected with C. bainae, based on PCR analyses of the blood of the infected dogs [42]. Specifically, 15 dogs were co-infected with C. bainae and Hepatozoon canis, and two dogs were infected with C. bainae, H. canis and Anaplasma platys [42]. Similar results have also been reported in southern Italy, where 58 C. bainae-infected

(See figure on next page.)

Fig. 4 Phylogenetic relationship of Cercopithifilaria spp. sequences detected in this study (in bold) and other filarioid nematodes available from GenBank based on a partial sequence of the cox 1 gene. Evolutionary analysis was conducted on 1000 bootstrap replications using a maximum likelihood method and Tamura-Nei model to model evolutionary rate differences among sites selected by best-fit model. A homologous sequence from Litosomoides yutajensis (GenBank accession number: AJ544869) was used as the outgroup 


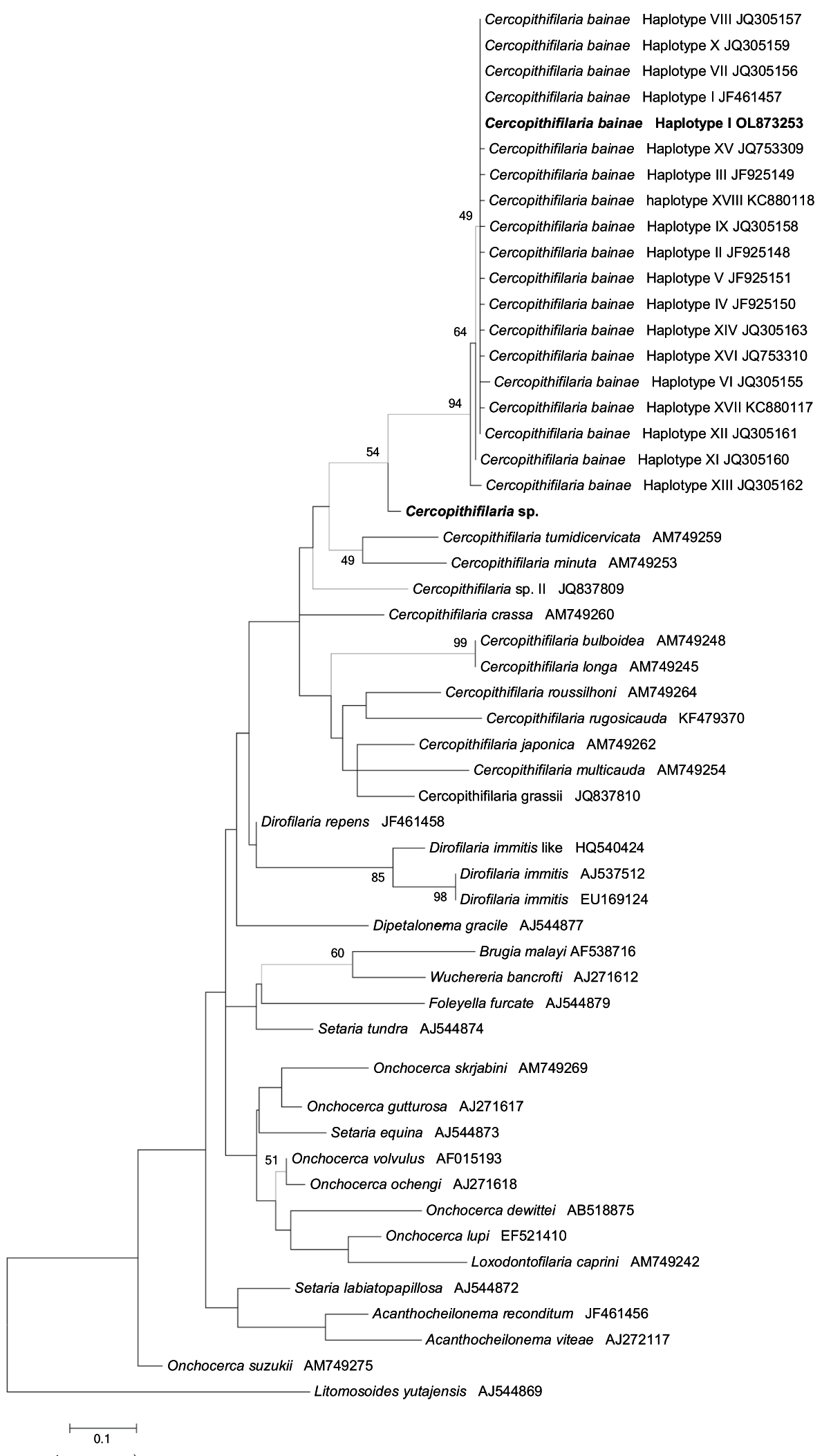

Fig. 4 (See legend on previous page.) 
Table 2 Cercopithifilaria infection rate in dogs included in the study according to geographical location and climatic parameters

\begin{tabular}{|c|c|c|c|c|c|c|}
\hline Study area & $\begin{array}{l}\text { Cercopithifiaria } \\
\text { infection rate }(\%)^{a}\end{array}$ & Coordinates & $\begin{array}{l}\text { Elevation above } \\
\text { sea level }(\mathrm{m})\end{array}$ & Climate & $\begin{array}{l}\text { Average annual } \\
\text { precipitation (mm) }\end{array}$ & $\begin{array}{l}\text { Average annual } \\
\text { temperature }\left({ }^{\circ} \mathrm{C}\right)\end{array}$ \\
\hline Kermanshah & 25 & $34.3277^{\circ} \mathrm{N}, 47.0778^{\circ} \mathrm{E}$ & 1358 & Warm and temperate & 437 & 13.3 \\
\hline Yazd & 8.7 & $31.8974^{\circ} \mathrm{N}, 54.3569^{\circ} \mathrm{E}$ & 1230 & Hot and arid & 55 & 18.9 \\
\hline Hamedan & 5.6 & $34.7989^{\circ} \mathrm{N}, 48.5150^{\circ} \mathrm{E}$ & 1823 & Cold semi-arid & 384 & 11.3 \\
\hline Amol & 3.7 & $36.4676^{\circ} \mathrm{N}, 52.3507^{\circ} \mathrm{E}$ & 96 & Mild and humid & 829 & 15.9 \\
\hline Esfahan & 3.7 & $32.6539^{\circ} \mathrm{N}, 51.6660^{\circ} \mathrm{E}$ & 1590 & Desert & 97 & 16.7 \\
\hline Khorramabad & 0 & $33.4647^{\circ} \mathrm{N}, 48.3390^{\circ} \mathrm{E}$ & 1147 & Mediterranean & 511 & 17.2 \\
\hline Ahvaz & 0 & $31.3183^{\circ} \mathrm{N}, 48.6706^{\circ} \mathrm{E}$ & 17 & Hot and humid & 210 & 24.9 \\
\hline
\end{tabular}

${ }^{a}$ (Number of biopsies positive for Cercopithifilaria bainae/total number of biopsies) $\times 100$

tick-borne pathogen, such as $H$. canis, A. platys and Babesia vogeli [43]. The detection of $C$. bainae microfilariae indicates a prior tick exposure and should stimulate testing for other tick-borne pathogens. However, it has not yet been demonstrated whether $C$. bainae infection adversely affects the dog's immune response to other pathogens [43].

Microfilariae of O. lupi were not detected in the examined samples, although $11.8 \%$ of the evaluated dogs showed mucosal discharges, conjunctivitis, keratitis or ulcers. Common ocular alterations of O. lupi in dogs are granulomatous mass on the scleral tissue, conjunctival swelling, keratitis, uveitis and mucopurulent discharge, as well as blindness [25]. Atypical migration of $O$. lupi in the larynx lumen of a dog has also been documented [44]. Cutaneous microfilariae of O. lupi have been reported in dogs and cats in Europe (e.g. Italy, Greece, Romania, Germany, Portugal, Spain, Switzerland), USA and Canada [45]. However, in areas outside of Europe and North America, such as Iran [24], Turkey [18, 22] and Tunisia [46], human cases of $O$. lupi are possibly associated with wild carnivores, which could play a role in the epidemiology of the infection [23].

In our study, the skin sediments of the examined cats and golden jackals tested negative for filarioids, probably due to the small sample size analysed. However, $O$. lupi has been previously reported in cats from Portugal and USA $[47,48]$. On the other hand, the role of C. aureus in the epidemiology of Cercopithifilaria spp. and Onchocerca spp. is not known [49], advocating further research to elucidate the role of these animals as sylvatic carriers or reservoir hosts of these parasites.

All of the C. bainae isolated in the present study belonged to haplotype I. In contrast, a high genetic variability for $C$. bainae has been reported in previous studies conducted in Italy, Spain and Greece [10, $26,50]$, suggesting a late introduction of the parasite from Europe. To date, 21 cox 1 haplotypes of C. bainae have been identified [14, 26, 50], with haplotype I being the most commonly detected in dogs in the Mediterranean basin [50], as well as in $R$. sanguineus s.l. ticks from Italy, Greece, Malaysia, South Africa and USA, $R$. turanicus from Italy and Rhipicephalus sp. I from Greece and Italy [14, 26, 41]. The finding of three sequences displaying a relatively low nucleotide similarity (93.4\%) with that of haplotype I (GenBank accession number: JF461457) is difficult to interpret, suggesting the existence of a different, as yet molecularly not characterised, species of Cercopithifilaria.

No information on the tick species circulating the microfilariae of Cercopithifilaria spp. in Iran is currently available. However, studies performed in Italy, Brazil, Spain, Portugal, Australia, Malaysia, Pakistan and South Africa reported C. bainae in different Rhipicephalus spp., indicating low specificity of these filarioids for ticks of this genus $[14,51,52]$. Therefore, further studies on dogs and tick vectors are needed to obtain a clearer picture of the geographical distribution of all three known Cercopithifilaria species infecting dogs in western Asia.

\section{Conclusions}

This study reports for the first time C. bainae infection in dogs from Iran, expanding current knowledge on the geographical distribution of this parasite. Further studies on the different wild canine species (e.g., jackal, fox, wolf) and Rhipicephalus spp. ticks present in the country are needed to better understand the epidemiology of Cercopithifilaria spp. in the study area. Additionally, considering the previous human reports of O. lupi in Iran, more investigations on dogs as hosts should be performed with a focus on this zoonotic nematode.

\section{Abbreviations}

BLAST: Basic Local Alignment Search Tool; Cl: Confidence interval; cox1: Cytochrome c oxidase subunit 1; ML: Maximum Likelihood; NaCl: Sodium chloride; NGO: Non-governmental organisation; s.l.: Sensu lato. 


\section{Supplementary Information}

The online version contains supplementary material available at https://doi. org/10.1186/s13071-021-05141-2.

Additional file 1: Video S1. Cercopithifilaria bainae microfilaria during the microscopic examination of a skin sediment ( $\times 400$ magnification).

\section{Acknowledgements}

We thank all who assisted us in sampling, with specials thanks to Aliasghar Bahari, Seyedmasoud Zolhavarieh and Alireza Nourian (Bu-Ali Sina University); Gholamreza Mowlavi, Omid Hosseininejad, Fatemeh Arabifar and Mahmood Khannejad (in Ahvaz); Shilan Lorestani (in Kermanshah); Faranak Rahmanpour (in Amol); and Seyed Ali Vaziri (in Yazd). This study was performed under the academic agreement between Bu-Ali Sina University (Iran) with University of Bari (Italy).

\section{Authors' contributions}

Conceptualisation: AS, DO. Methodology: AS, ZB, FN, GA, HN, AMS, SMM, SMS. Writing/preparation of original draft: AS, GA, ZB. Writing, review and editing: $A S, M A B S, D O$. Supervision, project administration and funding acquisition: AS, DO. All authors have read and approved the final version of the manuscript.

\section{Funding}

This study was supported financially by Bu-Ali Sina University (Grant number 1/1/28864 to AS) and Shiraz University of Medical Sciences (Grant number 17351 to SMS).

\section{Availability of data and materials}

All data generated or analysed during this study are included in this published article and its additional files.

\section{Declarations}

\section{Ethics approval and consent to participate}

Sampling in Hamedan, Kermanshah, Amol, Yazd and Ahvaz was approved by the Ethical Research Committee of Faculty of Veterinary Science, Bu-Ali Sina University. Sampling in Esfahan was approved by the Research Ethics Committee of Shiraz University of Medical Sciences (Approval ID: IR.SUMS. REC.1398.1112).

\section{Consent for publication}

Not applicable.

\section{Competing interests}

The authors declare that they have no competing interests.

\section{Author details}

'Department of Pathobiology, Faculty of Veterinary Science, Bu-Ali Sina University, 6517658978 Hamedan, Iran. ${ }^{2}$ Zoonotic Diseases Research Center, School of Public Health, Shahid Sadoughi University of Medical Sciences, 8915173160 Yazd, Iran. ${ }^{3}$ Department of Veterinary Medicine, University of Bari Aldo Moro, Str. prov. per Casamassima km 3, Valenzano, 70010 Bari, Italy. ${ }^{4}$ Department of Pathobiology, Faculty of Veterinary Medicine, Lorestan University, 6815144316 Khorramabad, Iran. ${ }^{5}$ Department of Parasitology and Mycology, School of Medicine, Shiraz University of Medical Sciences, Shiraz, Iran. ${ }^{6}$ Research Center of Basic Sciences in Infectious Diseases, Shiraz University of Medical Sciences, Shiraz, Iran.

Received: 30 October 2021 Accepted: 22 December 2021 Published online: 20 January 2022

\section{References}

1. Otranto D, Brianti E, Latrofa MS, Annoscia G, Weigl S, Lia RP, et al. On a Cercopithifilaria sp. transmitted by Rhipicephalus sanguineus: a neglected, but widespread filarioid of dogs. Parasit Vectors. 2012;5:1.
2. Mutafchiev Y, Dantas-Torres F, Giannelli A, Abramo F, Papadopoulos E, Cardoso L, et al. Redescription of Onchocerca lupi (Spirurida: Onchocercidae) with histopathological observations. Parasit Vectors. 2013;6:309.

3. Otranto D, Varcasia A, Solinas C, Scala A, Brianti E, Dantas-Torres F, et al. Redescription of Cercopithifilaria bainae Almeida \& Vicente, 1984 (Spirurida, Onchocercidae) from a dog in Sardinia, Italy. Parasit Vectors. 2013;6:132.

4. Bain O, Uni S, Takaoka H. A synthetic look at a twenty year old taxon, Cercopithifilaria; its probable evolution. In: 10th International Congress of Parasitology (ICOPA X), 4-9 August, 2002; Vancouver. p. 365-8.

5. Lefoulon E, Kuzmin Y, Plantard O, Mutafchiev Y, Otranto D, Martin C, et al. Redescription of Cercopithifilaria rugosicauda (Böhm \& Supperer, 1953) (Spirurida: Onchocercidae) of roe deer, with an emended diagnosis of the genus Cercopithifilaria and a genetic characterisation. Parasitol Int. 2014;63:808-16.

6. Maia C, Casero M, Annoscia G, Latrofa MS, Colella V, Pereira A, et al. Cercopithifilaria sp. II in Vulpes vulpes: new host affiliation for an enigmatic canine filarioid. Parasitol Res. 2017;116:441-3.

7. Boyd M, Santoro D, Craft WF, Ginn PE, Childress AL, Wellehan JF, et al. Dermatitis caused by autochthonous Cercopithifilaria bainae from a dog in Florida, USA: clinical, histological and parasitological diagnosis and treatment. Vet Dermatol. 2019;30:68-e20.

8. Otranto D, Brianti E, Abramo F, Gaglio G, Napoli E, Latrofa MS, et al. Cutaneous distribution and localization of Cercopithifilaria sp. microfilariae in dogs. Vet Parasitol. 2012;190:143-50.

9. Gabrielli S, Giannelli A, Brianti E, Dantas-Torres F, Bufalini M, Fraulo M, et al. Chronic polyarthritis associated to Cercopithifilaria bainae infection in a dog. Vet Parasitol. 2014;205:401-4

10. Otranto D, Brianti E, Dantas-Torres F, Miro G, Latrofa MS, Mutafchiev $Y$, et al. Species diversity of dermal microfilariae of the genus Cercopithifilaria infesting dogs in the Mediterranean region. Parasitology. 2013;140:99-108

11. Soares RL, Parolin LB, Mateus NLF, de Figueiredo GRD, Rodrigues VR, de Oliveira GG, et al. Giant cutaneous cyst in a dog infected by Cercopithifilaria bainae. Vet Parasitol Reg Stud Rep. 2020;20:100401.

12. Brianti E, Otranto D, Dantas-Torres F, Weigl S, Latrofa M, Gaglio G, et al. Rhipicephalus sanguineus (Ixodida, Ixodidae) as intermediate host of a canine neglected filarial species with dermal microfilariae. Vet Parasitol. 2012;183:330-7.

13. Ramos RAN, Giannelli A, Brianti E, Annoscia G, Cantacessi C, Dantas-Torres $F$, et al. Tick vectors of Cercopithifilaria bainae in dogs: Rhipicephalus sanguineus sensu lato versus /xodes ricinus. Parasitol Res. 2013;112:3013-7.

14. Latrofa MS, Dantas-Torres F, Giannelli A, Otranto D. Molecular detection of tick-borne pathogens in Rhipicephalus sanguineus group ticks. Ticks Tick Borne Dis. 2014;5:943-6.

15. Rojas A, Morales-Calvo F, Salant H, Otranto D, Baneth G. Zoonotic ocular onchocercosis by Onchocerca lupi. Yale J Biol Med. 2021;94:331-41.

16. Otranto D, Giannelli A, Scotty Trumble N, Chavkin M, Kennard G, Latrofa MS, et al. Clinical case presentation and a review of the literature of canine onchocercosis by Onchocerca lupi in the United States. Parasit Vectors. 2015:8:89.

17. Franchini D, Giannelli A, Di Paola G, Cortes H, Cardoso L, Lia RP, et al. mage diagnosis of zoonotic onchocercosis by Onchocerca lupi. Vet Parasitol. 2014;203:91-5

18. Otranto D, Sakru N, Testini G, Gürlü VP, Yakar K, Lia RP, et al. First evidence of human zoonotic infection by Onchocerca lupi (Spirurida, Onchocercidae). Am J Trop Med. 2011;84:55-8.

19. Otranto D, Colella V, Bezerra-Santos MA, Mendoza-Roldan JA, Cavalera MA, Pereira A, et al. Efficacy of a spot-on formulation containing moxidectin 2.5\%/imidacloprid $10 \%$ for the treatment of Cercopithifilaria spp. and Onchocerca lupi microfilariae in naturally infected dogs from Portugal. Parasit Vectors. 2021:14:199.

20. Cantey PT, Weeks J, Edwards M, Rao S, Ostovar GA, Dehority W, et al. The emergence of zoonotic Onchocerca lupi infection in the United States-a case-series. Clin Infect Dis. 2015;62:778-83.

21. Dudley RW, Smith C, Dishop M, Mirsky D, Handler MH, Rao S. A cervical spine mass caused by Onchocerca lupi. Lancet. 2015;386:1372.

22. Hasanreisoglu M, Halim MS, Latrofa MS, Mendoza-Roldan JA, Öğüt B, Yilmaz $M$, et al. A human case of Onchocerca lupi mimicking nodular scleritis. Am J Trop Med. 2021;105(6):1782-5. 
23. Otranto D, Deplazes P. Zoonotic nematodes of wild carnivores. Int J Parasitol Parasit Wildl. 2019;9:370-83.

24. Mowlavi G, Farzbod F, Kheirkhah A, Mobedi I, Bowman DD, Naddaf SR Human ocular onchocerciasis caused by Onchocerca lupi (Spirurida, Onchocercidae) in Iran. J Helminthol. 2014;88:250-5.

25. Otranto D, Dantas-Torres F, Giannelli A, Latrofa MS, Papadopoulos E, Cardoso L, et al. Zoonotic Onchocerca lupi infection in dogs, Greece and Portugal, 2011-2012. Emerg Infect Dis. 2013;19:2000-3.

26. Angelou A, Latrofa MS, Annoscia G, Symeonidou I, Theodoridis A, Polizopoulou ZS, et al. Cercopithifilaria species in dogs and ticks from Greece. Parasitol Res. 2020;119:3391-400.

27. Mosallanejad B, Alborzi A, Katvandi N. A survey on ectoparasite infestations in companion dogs of Ahvaz district, south-west of Iran. J Arthropod Borne Dis. 2012;6:70-8.

28. Ebrahimzade E, Fattahi R, Ahoo MB. Ectoparasites of stray dogs in Mazandaran, Gilan and Qazvin Provinces, north and center of Iran. Arthropod Borne Dis. 2016;10:366-71.

29. Ranjbar Bahadori S. Study of species diversity of animal ticks in Garmsar [in Persian with English abstract]. J Fac Vet Med University Tehran. 2003:58:11-4.

30. Mirani F, Yakhchali M, Naem S. A study on ectoparasites fauna of dogs in suburbs of Ghilanegharb, Kermanshah province, Iran [in Persian with English abstract]. J Fac Vet Med Univ Tehran. 2017;72:7-14.

31. Khazeni A, Telmadarraiy Z, Oshaghi MA, Mohebali M, Zarei Z, Abtahi SM. Molecular detection of Ehrlichia canis in ticks population collected on dogs in Meshkin-Shahr, Ardebil Province, Iran. J Biomed Eng. 2013;6:1-5.

32. Ghahvei Y, Mirzaei M, Hashemnia S, Golchin M, Kheirandish R, Uni S, et al. Scanning electron microscopy of Onchocerca fasciata (Filarioidea: Onchocercidae) adults, microfilariae and eggs with notes on histopathological findings in camels. Parasit Vectors. 2020;13:249.

33. Latrofa MS, Weigl S, Dantas-Torres F, Annoscia G, Traversa D, Brianti E, et al. A multiplex PCR for the simultaneous detection of species of filarioids infesting dogs. Acta Trop. 2012;122:150-4.

34. Tamura K, Nei M. Estimation of the number of nucleotide substitutions in the control region of mitochondrial DNA in humans and chimpanzees. Mol Biol Evol. 1993;10:512-26.

35. Nei M, Kumar S. Molecular evolution and phylogenetics. Oxford University Press; 2000.

36. Tamura K, Peterson D, Peterson N, Stecher G, Nei M, Kumar S. MEGA5: molecular evolutionary genetics analysis using maximum likelihood, evolutionary distance, and maximum parsimony methods. Mol Biol Evol. 2011:28:2731-9.

37. Solinas C, Varcasia A, Brianti E, Giannetto S, Pipia AP, Columbano N, et al. Cercopithifilaria spp. in dogs in Sardinia Island (Italy). Parasitol Res. 2014;113:675-9.

38. Cortes HC, Cardoso L, Giannelli A, Latrofa MS, Dantas-Torres F, Otranto D. Diversity of Cercopithifilaria species in dogs from Portugal. Parasit Vectors. 2014;7:261.

39. Ionică AM, D'Amico G, Mitková B, Kalmár Z, Annoscia G, Otranto D, et al. First report of Cercopithifilaria spp. in dogs from Eastern Europe with an overview of their geographic distribution in Europe. Parasitol Res. 2014;113:2761-4.

40. Ramos RAN, do Rêgo AGDO, de FariasFirmino ED, Ramos CADN, de Carvalho GA, Dantas-Torres F, et al. Filarioids infecting dogs in northeastern Brazil. Vet Parasitol. 2016;226:26-9.

41. Lineberry MW, Sundstrom KD, Little SE, Stayton EM, Allen KE. Detection of Cercopithifilaria bainae infection in shelter dogs and ticks in Oklahoma, USA. Parasit Vectors. 2020;13:216.

42. Iatta R, Sazmand A, Nguyen V-L, Nemati F, Bahiraei Z, Mazhar Ayaz M, et al. Vector-borne pathogens in dogs of different regions of Iran and Pakistan. Parasitol Res. 2021;120:4219-28.

43. Ramos RAN, Giannelli A, Lia RP, Brianti E, Tarallo VD, Breitshwerdt EB, et al. Incidence of Cercopithifilaria bainae in dogs and probability of coinfection with other tick-borne pathogens. PLoS ONE. 2014;9:e88198.

44. Alho AM, Cruz L, Coelho A, Martinho F, Mansinho M, Annoscia G, et al. Aberrant laryngeal location of Onchocerca lupi in a dog. Parasitol Int. 2016;65:218-20

45. Verocai GG, Conboy G, Lejeune M, Marron F, Hanna P, MacDonald E, et al. Onchocerca lupi nematodes in dogs exported from the United States into Canada. Emerg Infect Dis. 2016;22:1477.
46. Otranto D, Dantas-Torres F, Cebeci Z, Yeniad B, Buyukbabani N, Boral OB, et al. Human ocular filariasis: further evidence on the zoonotic role of Onchocerca lupi. Parasit Vectors. 2012;5:84

47. Maia C, Annoscia G, Latrofa MS, Pereira A, Giannelli A, Pedroso L, et al. Onchocerca lupi nematode in cat, Portugal. Emerg Infect Dis. 2015;21:2252

48. Labelle AL, Daniels JB, Dix M, Labelle P. Onchocerca lupi causing ocular disease in two cats. Vet Ophthalmol. 2011;14:105-10.

49. Gherman CM, Mihalca AD. A synoptic overview of golden jackal parasites reveals high diversity of species. Parasit Vectors. 2017;10:419.

50. Otranto D, Latrofa MS, Brianti E, Annoscia G, Parisi A, Dantas-Torres F, et al. An assessment of genetic variability in the mitochondrial cytochrome $c$ oxidase subunit 1 gene of Cercopithifilaria sp. (Spirurida, Onchocercidae) from dog and Rhipicephalus sanguineus populations. Mol Cell Probes. 2012;26:81-9.

51. Santos MAB, de Macedo LO, Otranto D, Ramos CAN, Rêgo AGO, Giannelli A, et al. Screening of Cercopithifilaria bainae and Hepatozoon canis in ticks collected from dogs of Northeastern Brazil. Acta Parasitol. 2018;63:605-8.

52. Santos MAB, de Souza IB, de Macedo LO, Ramos CAN, Rego AGO, Alves $L C$, et al. Cercopithifilaria bainae in Rhipicephalus sanguineus sensu lato ticks from dogs in Brazil. Ticks Tick Borne Dis. 2017;8:623-5.

\section{Publisher's Note}

Springer Nature remains neutral with regard to jurisdictional claims in published maps and institutional affiliations.

Ready to submit your research? Choose BMC and benefit from

- fast, convenient online submission

- thorough peer review by experienced researchers in your field

- rapid publication on acceptance

- support for research data, including large and complex data types

- gold Open Access which fosters wider collaboration and increased citations

- maximum visibility for your research: over 100M website views per year

At BMC, research is always in progress.

Learn more biomedcentral.com/submissions 